\title{
Intermédialités
}

Histoire et théorie des arts, des lettres et des techniques

Intermediality

History and Theory of the Arts, Literature and Technologies

\section{Du document aux flux : sites et temporalité(s) de quelques oeuvres d'art}

\section{Suzanne Paquet}

Numéro 33, printemps 2019

restituer (le temps)

rendering (time)

URI : https://id.erudit.org/iderudit/1065015ar

DOI : https://doi.org/10.7202/1065015ar

Aller au sommaire du numéro

Éditeur(s)

Revue intermédialités

ISSN

1920-3136 (numérique)

Découvrir la revue

Citer cet article

Paquet, S. (2019). Du document aux flux : sites et temporalité(s) de quelques oeuvres d'art. Intermédialités / Intermediality, (33).

https://doi.org/10.7202/1065015ar
Résumé de l'article

Cet article étudie, à partir de l'image (photographique), les modes d'être de certaines oeuvres d'art, pour lesquelles il n'y a plus de médium unique, mais tout un réseau de gestes, d'images, de résonances et de reprises, circulant et se multipliant, composant ainsi une chronogéographie inédite. Ce sont les questions de leur médialité et des flux sociotechniques contribuant à faire de ces propositions artistiques des entités en réseaux, ainsi que leur temporalité particulière se conjuguant au présent et par la présence, qui sont examinées et exemplifiées à partir des oeuvres Spiral Jetty (1970), de Robert Smithson, Sun Tunnels (1976), de Nancy Holt, et Virtually There (2009-), d'Andreas Rutkauskas. 


\title{
Du document aux flux : sites et temporalité(s) de quelques œuvres d'art
}

\author{
Suzanne Paquet
}

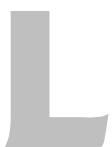

a photographie, ou plutôt les photographies, se constituent en objets temporels, réputés traverser les époques, pour restituer lieux et moments fixés dans des ailleurs et dans des temps autres. Les instants saisis par la photographie tiendraient d'une «conjonction illogique entre l'ici et l'autrefois ${ }^{\mathrm{I}} \gg$ selon Roland Barthes et formeraient aussi en quelque sorte un inframince, un flottement entre « plus jamais » et « pas encore », ajoute Thierry de Duve (« ... it is the sudden vanishing of the present tense, splitting into the contradiction of being simultaneously too late and too early, that is properly unbearable $\left.{ }^{2} \gg\right)$. Documents témoignant du passé, consultés au présent (des présents qui se succéderont et s'accumuleront si la photographie a une longue vie) et assurant un certain futur, celui du souvenir, aux choses figées par l'action du médium, les images photographiques induisent une forme d'anachronisme ( « jouer sur la longue durée et jouer sur l'instant présent », les « deux tableaux du temps ${ }^{3} »$ auxquels l'empreinte, que l'on a longtemps associée à la photographie, renvoie) ou de contemporanéité :

\footnotetext{
${ }^{\mathrm{I}}$ Roland Barthes, « Rhétorique de l'image », Communications, $\mathrm{n}^{\circ} 4$, 1964 , p. 47. Italique de l'auteur. En ligne: https://www.persee.fr/doc/comm_0588-80I8_I964_num_4_I_IO27 (consulté le 28 mars 2019).

${ }^{2}$ Thierry de Duve, «Time Exposure and Snapshot: The Photograph as Paradox », October, vol. 5 , été 1978 , p. I2I.

${ }^{3}$ Georges Didi-Huberman, La ressemblance par contact. Archéologie, anachronisme et modernité de l'empreinte, Paris, Éditions de Minuit, 2008, p. 23.
} 
Comprenez bien que le rendez-vous dont il s'agit dans la contemporanéité ne se situe pas seulement dans le temps chronologique: il est, dans le temps chronologique, quelque chose qui le travaille de l'intérieur et le transforme. Et cette urgence, c'est l'inactualité, l'anachronisme qui permet de saisir notre temps sous la forme d'un « trop tôt » qui est aussi un « trop tard », d'un « déjà » qui est aussi un « pas encore ${ }^{4} \gg$.

L'image photographique a pris une importance de plus en plus grande dans toute la culture visuelle occidentale (et au-delà), à travers ses quasi-deux siècles d'existence. Elle accompagne les rites de passage et divers moments de la vie quotidienne, elle montre encore les horreurs et les catastrophes, se fait aussi le véhicule de la notoriété et, surtout, accompagne l'art de diverses façons, ce qui m'intéressera plus particulièrement dans cet essai. Témoin et preuve, c'est sa légèreté, sa reproductibilité et sa grande mobilité qui en ont forgé le caractère incontournable. Depuis son avènement, elle voyage, traverse océans et continents, supporte les imaginaires géographiques et remplace les souvenirs par des images figées mais voyageuses; tout ceci formant une spatiotemporalité unique, encore agissante aujourd'hui, les images photographiques se multipliant, circulant et s'accumulant en nombre stupéfiant grâce notamment aux médias sociaux et à l'action des amateurs.

Dans les années 1960 et 1970, plus sûrement en régime postmoderne qu'à l'ère moderne où elle était pourtant déjà très présente, la photographie envahit fermement l'art contemporain's. On eût dit que l'art ne pouvait plus s'envisager sans elle, entre l'appropriation et la citation par reproduction mécanique (pictures generation, notamment), l'usage de photographies banales et sans qualité, sauf pour une certaine valeur descriptive ou de tenant-lieu (l'art conceptuel), et l'omniprésence du document (land art, body art, performance, etc.). De plus, comme le soulignait Rosalind Krauss, quelque chose de photographique, une qualité indicielle, pouvait décrire tout l'art du temps ${ }^{6}$.

${ }_{4}^{4}$ Giorgio Agamben, Qu'est-ce que le contemporain?, trad. par Maxime Rovere, Paris, Rivages poche/Petite bibliothèque, 2008, p. 25-26.

s Douglas Crimp, «The Photographic Activity of Postmodernism », On the Museum's Ruins [1993], Cambridge, MA, MIT Press, 2000, p. I08-I25.

${ }^{6}$ «Des procédures, des opérations qui ressortaient elles-mêmes à l'ordre de la trace (ouverte, frayée à même le sol, dans le cas du land art) ou de l'empreinte (laissée par un corps, ou qu'il exhibait, s'agissant du body art), et dans lesquelles Rosalind Krauss a su reconnaître l'emprise du modèle photographique. » Hubert Damisch, « À partir de la photographie », préface à Rosalind Krauss, Le photographique. Pour une théorie des écarts, Paris, Macula, 1990, p. 7 . 
Ainsi, la réflexion que je compte mener sur les sites et les temps de certaines œuvres d'art s'arrêtera d'abord sur le cas du land art, qui semble avoir créé toute une géographie de l'art, grâce aux sites choisis pour ses œuvres, à leur caractère inamovible et à la grande production de documentation photographique qui les accompagna; documentation forcément appelée par leur qualité strictement in situ et qui commandait une certaine mise en mouvement. J'observerai plus particulièrement la Spiral Jetty (1970), de Robert Smithson, et les Sun Tunnels (1976), de Nancy Holt; on comprendra pourquoi ils ont été, et restent, exemplaires à cet égard. La photographie se faisait donc là spatiale, bien qu'elle ait aussi contribué à la mémoire ou à la (sur)vie des œuvres, suivant cette temporalité qui lui est propre. Par la suite, tout en continuant de réfléchir le land art dans ses formes de mobilité et de réception actuelles, je ferai appel à des œuvres plus récentes. Une série photographique de l'artiste canadien Andreas Rutkauskas, Virtually There (2009 à ce jour)7, sera examinée afin de vérifier si spatialité et temporalité y interagissent différemment, l'hypothèse étant qu'à l'ère de l'hyperconnexion et des flux, la spatiotemporalité de l'image photographique $-\mathrm{du}$ document ou de l'œuvre d'art - pourrait jusqu'à un certain point s'envisager différemment, la géographie des images devenue une chronogéographie conférant aux œuvres une temporalité plus complexe et plus riche en même temps qu'une spatialité pour ainsi dire augmentée. Dans cette perspective, il faudra définir ce que pourrait être une chronogéographie adaptée à l'art, qui serait quelque peu différente de ce que l'on entend généralement par «chronogeography » ou «time geography ${ }^{8} »$. Là où il n’y a plus de médium singulier, mais tout un réseau de gestes, d'images, de résonances et de reprises, circulant et se multipliant dans le temps comme dans l'espace, dans les flux constants où elle s'inscrit et qu'elle-même compose, la photographie prend une qualité nouvelle.

\footnotetext{
7 Voir la présentation publiée sur le site de l'artiste : http://www.andreasrutkauskas.com/virtually-there (consulté le I8 mars 2019).

${ }^{8}$ Don N. Parkes et Nigel J. Thrift, Times, Spaces and Places: A Chronogeographic Perspective, New York, John Wiley and Sons, 1980; Jon May et Nigel J. Thrift (eds.), Timespace. Geographies of Temporality, London and New York, Routledge, 20oI.
} 


\section{CHRONOGÉOGRAPHIE(S)}

Les tenants d'une chronogéographie ou d'une «géographie temporelle» (traduction possible de time geography, qu'en France on a tendance à ne pas traduire ${ }^{9}$ ) postulent que les activités et les expériences, des individus comme des collectivités, sont sujettes au pouvoir du temps tout aussi bien qu'aux pouvoirs spatiaux ${ }^{10}$.Il s'agit donc, dans l'analyse géographique des activités et des actes humains, de ne pas séparer temps et espace, mais de les considérer comme inextricablement liés". Cela peut s'avérer très pertinent pour certains sujets touchant la géographie et l'art, le paysage notamment, puisqu'il est manifestement façonné par le temps, le temps qui passe, altérant lieux et points de vue, et le temps du regardeur, entendu comme temps pris pour la contemplation ou comme situation et moment historique particuliers. De même, pour un support mobile comme la photographie, la prise en compte des aires géographiques des déplacements, tout comme de la chronologie, est importante. Certains sites et lieux, en retour, peuvent déterminer des temporalités caractéristiques. Les « non-lieux », ces espaces de transit ainsi identifiés par Marc Augér ${ }^{2}$, pourraient aisément s'analyser de cette façon : comme en suspens, le non-lieu suppose ou représente un emplacement singulier, en quelque sorte atemporel. Dans les aéroports et les gares, on attend, dans un entre-deux où le temps semble ne pas exister ou se refermer sur lui-même, de changer de lieu; et dans les avions et les trains dans lesquels finalement on se déplace (dé-place), alors qu'on a l'impression d'être nulle part et hors du temps, des heures sont éludées, disparaissent et réapparaissent - ou pas, suivant le sens dans lequel on se meut. Le passage de la ligne de changement de date, par exemple, donne lieu à ce type d'expérience insolite.

La spatiotemporalité des sociétés, à partir du milieu du $19^{\mathrm{e}}$ siècle devient tout spécialement digne d'être examinée, suivant John May et Nigel J. Thrift ${ }^{13}$, car il y aurait là un moment de rupture ou de grands changements sociaux et géographiques causés

${ }^{9}$ Voir, par exemple, Thomas Thevenin, Transport -Espace -Temps: regard croisé entre time geography et géobistoire, Habilitation à diriger des recherches, Université de Bourgogne, 2010.

Io Yi-Fu Tuan, «A review of Time, Spaces and Places: A Chronogeographic Perspective », Annals of the Association of American Geographers, vol. 7I, nº 2, juin 198I, p. 293.

${ }^{\text {II }}$ May et Thrift, $200 I$.

${ }^{12}$ Marc Augé, Non-lieux: introduction à une anthropologie de la surmodernité, Paris, Éditions du Seuil, 1992.

${ }^{13}$ May et Thrift, 20oI. 
par des avancées techniques, ce que John Urry ${ }^{14}$ a lui aussi finement décrit. Dans les trois derniers quarts de ce siècle, on voit en effet apparaître la standardisation et la mécanisation à grande échelle du temps, les transports rapides - le train en particulier - et de nouveaux moyens de communication, le télégraphe puis le téléphone, et ainsi de suite, tous des développements liés à l'industrialisation et à l'urbanisation, auxquels il faut évidemment ajouter la photographie. Ces techniques, réunies, marquent une accélération du temps, induisent des sensations d'ubiquité ou de simultanéité inconnues jusqu'alors, ce dont l'art se fera le témoin - du réalisme jusqu'au cubisme et au futurisme, en passant par l'impressionnisme et Dada. La photographie - et, ultimement, les autres modes d'enregistrement - permet une sorte de feuilletage du temps : elle fixe et rend durable et consultable la réalité d'instants révolus. Il y aurait donc, par l'accumulation de toutes ces nouveautés, une « compression radicale des horizons spatiaux et temporels ${ }^{\mathrm{Is}}$ » et à la fin du $19^{\mathrm{e}}$ siècle se produiraient « simultanément un rétrécissement du monde et son élargissement, alors que les gens prennent conscience d'événements arrivés dans des parties du monde de plus en plus lointaines ${ }^{16} \gg$. À cet égard, le début du $2 \mathrm{I}^{\mathrm{e}}$ siècle serait, symétriquement, un autre moment fort où temps et espace, ensemble et à la fois, se rétrécissent et s'élargissent. La technique est de première importance lorsqu'il s'agit de réfléchir une chronogéographie. Généralement utile pour la mesure et l'analyse des déplacements et des activités humaines, souvent quotidiennes ${ }^{17}$, celle-ci est plutôt appliquée ici à des travaux artistiques imbriqués dans des réseaux complexes, sociotechniques, où interagissent objets, technologies et (agents) humains.

May et Thrift signalent également que des instruments qui semblent avoir été conçus pour marquer le passage du temps tendent à altérer le sens de l'espace; la télévision et le magnétoscope, par lesquels se créent de vastes «broadcasting communit[ies] », en seraient l'illustration ${ }^{18}$. Il y a là un phénomène correspondant au

${ }^{14}$ John Urry, The Tourist Gaze: Leisure and Travel in Contemporary Societies [1990], London, Sage Publications, 2002.

${ }^{\text {Is }}$ May et Thrift, 20oI, p. 7. Notre traduction.

${ }^{16}$ Ibid., p. 8. Notre traduction.

${ }^{17}$ Thévenin, 2010.

${ }^{18}$ May et Thrift, 20oI, p. 4. Les récents travaux de Marta Boni montrent l'importance, encore aujourd'hui, de ces communautés ou réseaux. Voir notamment « Des téléspectateurs géolocalisés. Traces de la réception et construction de l'espace à l'ère du numérique », Sens public, décembre 20I7, http://sens-public.org/articleı283.html (consulté le I8 mars 2019). 
«village global » autrefois rêvé par McLuhan ${ }^{19}$, aujourd'hui imaginé à travers Facebook ou Instagram. Déjà, dans les années 1960, une forme de réception collective et le fait d'être mis en relation par un intérêt partagé pour les séries télévisées ou tout autre objet temporel du genre, simultanément ou en différé, dans des endroits souvent très éloignés les uns des autres, suppose une chronogéographie en quelque sorte communautaire, les individus étant liés, en réseaux spatiotemporels, par des images techniques. La photographie, première image véritablement technique ${ }^{20}$, fut en effet très vite génératrice de communautés : on se regroupait autour de l'album de famille, on se rassemblait aussi pour faire de la prise de vues en certains endroits, comme on se réunit aujourd'hui autour de thématiques et de mots-clics (tags et hashtags) sur Flickr, Instagram ou Snapchat ${ }^{21}$. Outre cet aspect communautaire, la photographie, historiquement, fut surtout une technique permettant d'accumuler de petits morceaux de temps et d'espace, sous forme d'images, et de les faire circuler. Le $19^{\mathrm{e}}$ siècle voit en effet naître la pratique populaire du voyage en fauteuil et celle de l'accumulation d'images peu coûteuses : cabinets de stéréogrammes, cartes de visite, cartes postales... Le monde sera dorénavant connu et collectionné d'abord en images, et ce, tout aussi bien par les individus que par les États et les empires :

L'inventaire a débuté en I839 et depuis cette date, il n'est pratiquement pas une seule chose, semble-t-il, qui n'ait été photographiée. Cette boulimie même de l'œil photographique change les conditions de détention dans la caverne, notre monde. En nous enseignant un nouveau code visuel, les photographies modifient et élargissent notre idée de ce qui mérite d'être regardé et de ce que nous avons le droit d'observer. Elles constituent une grammaire et, ce qui est encore plus important, une éthique du regard. Enfin, le résultat le plus monumental de l'entreprise photographique est de nous donner le sentiment que le monde entier peut tenir dans notre tête, sous la forme d'une anthologie d'images.

19 Marshall McLuhan, The Gutenberg Galaxy. The Making of Typographic Man, Toronto, University of Toronto Press, 1962, p. 3 I.

${ }^{20}$ Vilém Flusser, Pour une philosophie de la photographie, trad. par Jean Mouchard, Saulxures, Circé, 1996; Vilém Flusser, Into the Universe of Technical Images, trad. par Nancy Ann Roth, Minneapolis, University of Minnesota Press, 2011.

${ }^{21}$ Réseaux sociaux basés sur la photographie, dont Flickr est le plus ancien (2004) et Snapchat, le plus récent (20II). 
Collectionner les photographies, c'est collectionner le monde. La lumière des films et des émissions de télévision illumine les murs, vacille et s'éteint; mais avec les photographies, l'image devient aussi objet, un objet léger, bon marché à produire, facile à transporter, à accumuler, à stocker ${ }^{22}$.

L'art n'échappera pas à cette nécessité d'inventaire, de mobilité et d'ubiquité, ce dont Benjamin fait le constat en 1935 dans son incontournable texte ${ }^{23}$ et que des chroniqueurs du $19^{\mathrm{e}}$ siècle avaient déjà célébré : « il [le daguerréotype] est destiné à populariser chez nous, et à peu de frais, les plus belles ouvres des $\operatorname{arts}{ }^{24} \gg$. La reproduction photographique des œuvres d'art se répand donc très vite et devient courante dès avant le début du $2 \mathrm{O}^{\mathrm{e}}$ siècle. Et le land art, arrivant dans sa seconde moitié, tracera, comme je le signalais plus haut, une nouvelle spatialité pour les œuvres, allant au-delà de la seule diffusion des reproductions. L'usage de documents et l'idée d'une certaine «dématérialisation» de l'art se répandent d'ailleurs dans les années 1960, simultanément à ces « broadcasting communities » dont parlent May et Thrift et à l'arrivée de la notion de village global ${ }^{25}$. C'est précisément cet aspect spatial, ou géographique, qui différencie les travaux des land artists de ceux de leurs contemporains faisant dans l'art conceptuel ou la performance : non seulement le document devient l'œuvre ou assure sa visibilité et sa survivance, mais il induit un double déplacement.

Comme les œuvres d'art conceptuel ou les performances, les œuvres du land art étatsunien devaient en effet s'accompagner d'une documentation, qui assurait en grande partie leur réception, en différé. Les idées, les actions et les performances étaient enregistrées par des images qui elles-mêmes étaient exposées dans les institutions, traces ou témoignages essentiels pour faire connaître les œuvres. Toutefois, la documentation

${ }^{22}$ Susan Sontag, Sur la photographie, trad. par Gérard-Henri Durand et Guy Durand, Paris, Éditions du Seuil, coll. « Io|I8 », 1983, p. 15-16.

${ }^{23}$ Walter Benjamin, «L'œuvre d'art à l'ère de sa reproductibilité technique », CFuvres III, trad. par Maurice de Gandillac et révisé par Rainer Rochlitz, Paris, Gallimard, coll. «Folio Essais », 2000, p. 67-II3.

${ }^{24}$ Jules Janin, «Le daguerréotype », paru dans L'Artiste en 1839, repris dans André Rouillé, La photographie en France. Textes E\& controverses: une anthologie 1816-1871, Paris, Macula, I989, p. 50.

${ }^{25}$ Lucy Lippard et John Chandler sont les premiers à avoir émis l'idée de dématérialisation des œuvres d'art, que Lippard développera dans un premier temps seule, dans Six Years: The Dematerialization of the Art Object from 1966 to 1972, Berkeley et Los Angeles, CA, University of California Press; ils s'appuient manifestement sur les théories de McLuhan pour énoncer la leur. Lucy Lippard et John Chandler, «The Dematerialization of Art », Art International, vol. I2 $\mathrm{n}^{\circ} 2$, février 1968, p. 3I-36. 
$\mathrm{du}$ land art suggérait d'autres horizons, ceux du voyage. Car, contrairement aux performances ou aux actions minuscules ou fugitives des conceptuels, les travaux des land artists existaient, et ont continué d'exister, matériellement, solidement ancrés quelque part dans le territoire; les documents leur permettaient simplement de s'expatrier, pour employer le beau mot d'Anne Cauquelin ${ }^{26}$, vers les musées et les galeries, où il était plus aisé de les voir que dans leur manifestation in situ. Prenons l'exemple de la Spiral Jetty, de Robert Smithson. Construite directement dans le Great Salt Lake, près de Rozel Point en Utah, cette œuvre monumentale (voir la figure I) y est toujours. Smithson avait conçu, dès sa construction en 1970, un film (The Spiral Jetty, couleur, 32 minutes) et une série de photographies, Stills from the Film Spiral Jetty, pouvant être exposés dans les institutions et même vendus. Ces « documents », selon l'artiste lui-même, faisaient partie intégrante de l'œuvre Spiral Jetty, qui devenait, pour ainsi dire, une œuvre en réseau :

Since he felt that many significant experiences in the twentieth century are vicarious ones available through secondary media such as film, video, and essays, Smithson decided to make secondary primary in Spiral Jetty. The Earthwork, then, does not consist merely of the spiraling causeway in Utah: the entire piece comprises the several works of art that frame the original Jetty ${ }^{27}$.

De plus, film et série photographique, intégrant des cartes et autres indices, pouvaient permettre d'aller visiter le site de la grande ouvre, en Utah, suivant le principe de site/non-site établi par Smithson et par lequel une œuvre appelée «nonsite», constituée de prélèvements (photographiques ou de matériaux, indifféremment), pouvait se substituer à son site de provenance, tout en étant une invitation à s'y rendre. Les images et le film remplissaient donc des fonctions temporelles et spatiales. Peu de gens, à l'époque, ont risqué le voyage vers le site lui-même, d'autant que la Spiral Jetty fut submergée dans les eaux du lac salé, de 1972 jusqu'au début des années 2000. L'œuvre resta toutefois visible, traversant ainsi les décennies grâce à ses images.

\footnotetext{
${ }^{26}$ Anne Cauquelin, Le site et le paysage, Paris, Presses universitaires de France, 2002, p. 156.
}

${ }^{27}$ Robert Hobbs, Robert Smithson: Sculpture, Ithaca, Cornell University Press, 198I, p. 17. 
DU DOCUMENT AUX FLUX :

SITES ET TEMPORALITÉ(S) DE QUELQUES CEUVRES D’ART

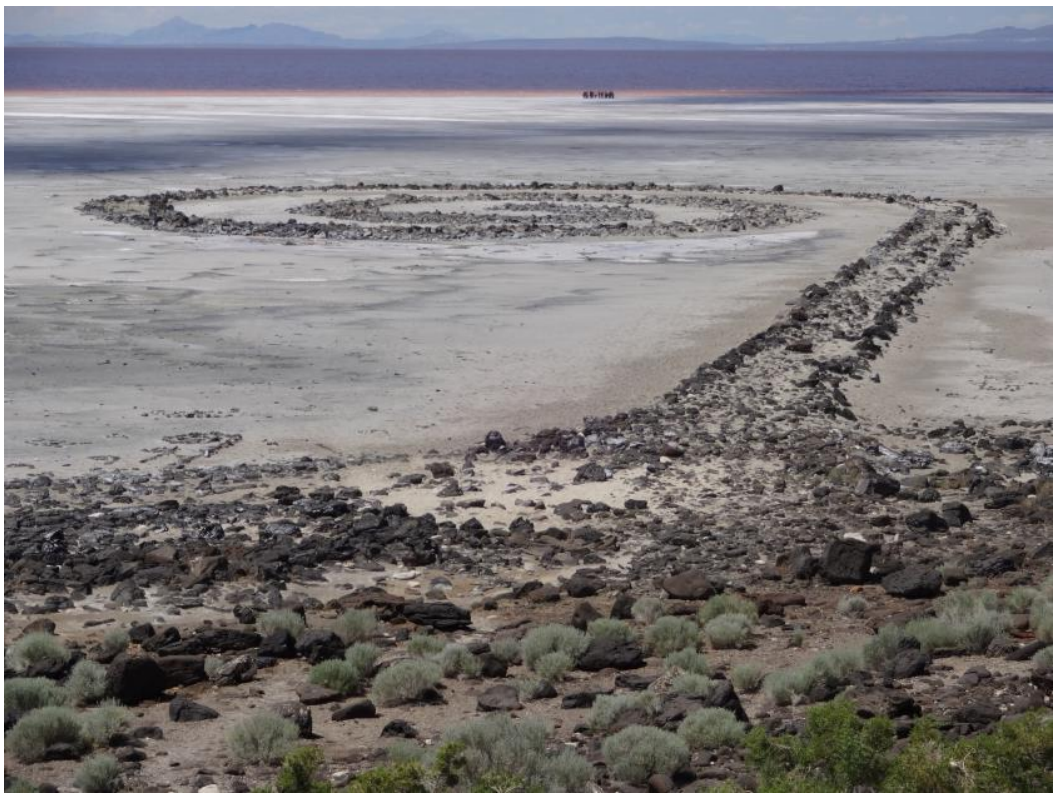

Figure I : Robert Smithson, Spiral Jetty, Great Salt Lake, Utah, 1970. Photographie: Suzanne Paquet.

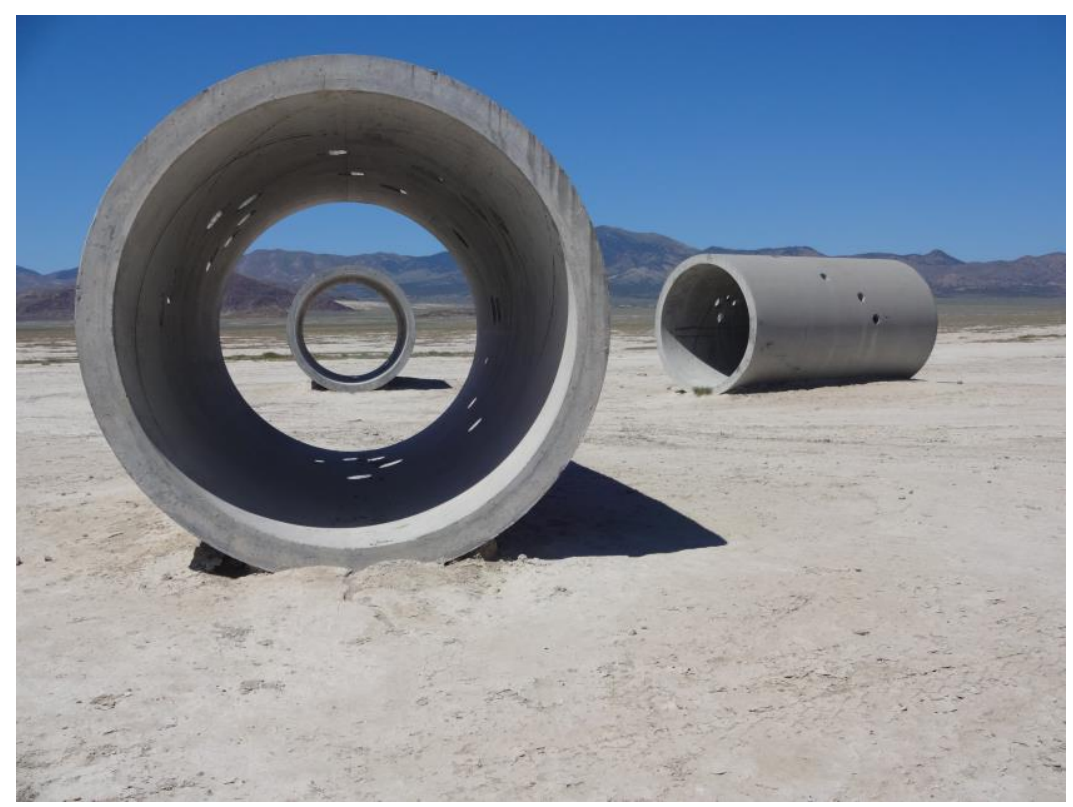

Figure 2 : Nancy Holt, Sun Tunnels, Great Basin Desert, Utah, 1973-1976. Photographie : Suzanne Paquet. 
Les Sun Tunnels (1973-1976), de Nancy Holt, furent installés dans le Great Basin Desert, aussi en Utah, tout près d'un village quasi fantôme nommé Lucin. Depuis, ils défient le temps, pratiquement inchangés. Ces quatre buses de béton (voir la figure 2), à leur façon, ont été conçues par Holt comme des objets temporels : bien fixées dans le désert, elles sont orientées de façon que des phénomènes naturels ayant, depuis des millénaires, influé sur la conception du temps des humains, y soient rendus parfaitement visibles. Les quatre tuyaux sont en effet disposés pour que le soleil, à son lever aux solstices d'hiver et d'été, s'y encadre exactement. Tout comme la Spiral Jetty, les Sun Tunnels ont longtemps veillé, seuls, sur leur portion de désert, visités seulement parfois par quelque historienne de l'art ou par des adorateurs du soleil qui $s^{\prime} y$ rendent lors des solstices ${ }^{28}$. Eux aussi ont survécu grâce à des photographies, bien que Holt n'ait pas privilégié l'idée que les documents puissent aussi être ou faire partie de l'œuvre : selon elle, l'expérience du site ne pouvait être remplacée par quoi que ce soit ${ }^{29}$. La notoriété de l'œuvre a tout de même été sauvegardée par les images, et ce sont bien elles qui ont suscité le désir de s'y rendre, suivant une géographie, un double parcours, pour ainsi dire touristique : l'image provoque le désir d'y aller voir, d'y faire «s photo $^{30} »$ qui, à son tour, donnera à d'autres l'envie d'y aller voir.

\section{MOBILITÉS (PHOTOGRAPHIQUES)}

Les déplacements induits par les œuvres de land art sont largement pratiqués aujourd'hui, certainement plus, d'ailleurs, que dans les années i97o. Ce développement chronogéographique tient très certainement à l'accélération de la mobilité produite par l'expansion des technologies numériques. Après une sorte d'éclipse historique, le land art a connu une recrudescence d'intérêt à partir du début des années 200o, qui ne se dément pas depuis. À tel point que les œuvres sont en voie de devenir des destinations ou des sites populaires et intemporels, ou figés dans le temps, comme si elles étaient elles-mêmes désormais des photographies que l'on irait visiter $^{3 \mathrm{I}}$. On pourrait même soupçonner que ces œuvres soient devenues des lieux de

\footnotetext{
${ }^{28}$ Voir à ce sujet Iris Amizlev, Land Art: Layers of Memory, the Uses of Prehistoric References in Land Art, thèse de doctorat, Université de Montréal, 1999.

${ }^{29}$ Nancy Holt, «Sun Tunnels », Artforum, avril 1977, p. 37.

${ }^{30}$ Marshall McLuhan, « La photographie. Le bordel imaginaire », Pour comprendre les médias. Les prolongements technologiques de l’homme [1968], trad. par Jean Paré, Montréal, Bibliothèque québécoise, 1993. L'italique est de l'auteur.

${ }^{31}$ Gilles Tiberghien, cherchant les Sun Tunnels: «J'arrive enfin sur le site avec un peu le sentiment de pénétrer dans une photographie mais pas du bon côté ». Gilles Tiberghien, Land Art Travelling, Valence, École régionale des Beaux-arts, 1996, p. 4I.
} 
pure photogénie, comme par exemple les chutes du Niagara, puisque l'on va depuis peu y faire des photographies de mariage (voir la figure 3)...

\section{Engagement Photos At The Spiral Jetty: Mallory + Christian}

REAL WEDDINGS // September 12, 2014

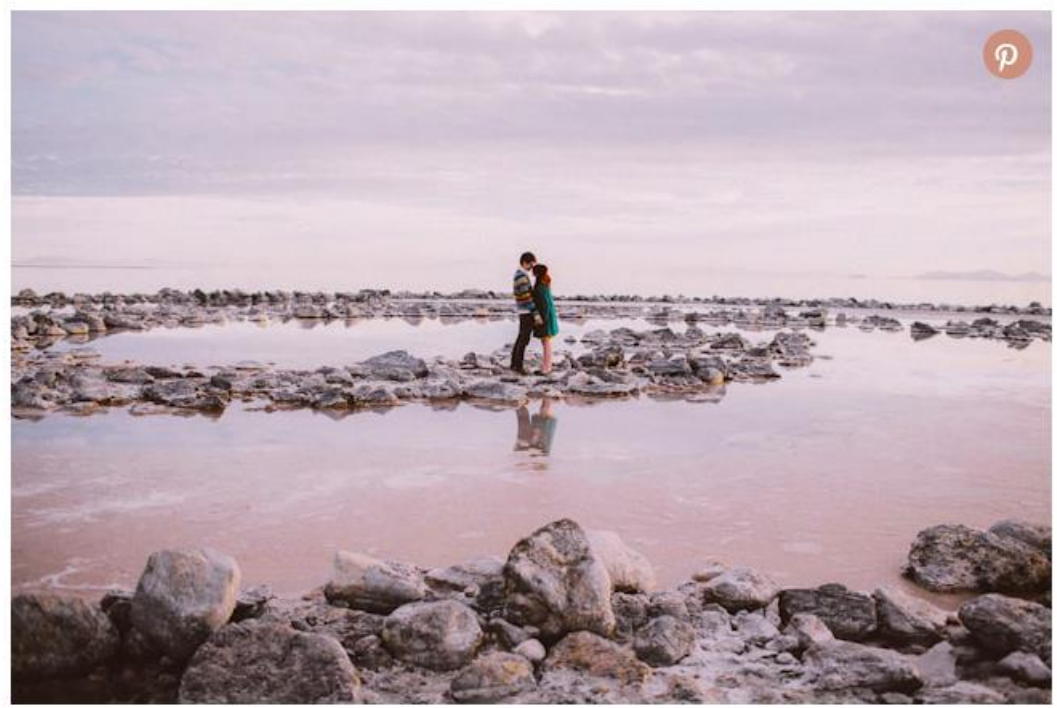

Figure 3: Photo de mariage à la Spiral Jetty. Capture d'écran : https://greenweddingshoes.com/engagement-photos-at-the-spiral-jetty/.

Au temps du Web participatif, chacun.e est devenu photographe, chacun.e documente abondamment sa propre vie. Nous sommes à l'ère de l'amateur ${ }^{32}$, plusieurs cherchant et pouvant parfois trouver à vendre leurs photographies, et chacun.e pouvant devenir prescriptrice ou « influenceur » grâce aux photographies diffusées : beaucoup de célébrités instantanées ont vu le jour par Instagram par exemple, le phénomène étant social et communicationnel tout en étant essentiellement photographique. Je note au passage que, bien que l'on puisse croire qu'avec l'arrivée du numérique, la photographie ne soit plus un médium en soi, dans la mesure où tout ce qui est écrit, image, son, etc., semble aujourd'hui fondu dans le

${ }^{32}$ Patrice Flichy, Le sacre de l'amateur. Sociologie des passions ordinaires à l'ère numérique, Paris, Éditions du Seuil, coll. « République des idées », 2 oIo. 
grand code binaire, elle a gardé toute la spécificité de ses usages sociaux; une relecture de Bourdieu est convaincante à cet égard ${ }^{33}$. Comme le résume Michelle Henning, « La photographie, alors, ne devrait pas tant être considérée comme un médium, mais bien comme une pratique sociale faisant usage de divers matériaux et divers moyens ${ }^{34} \gg$. Par ailleurs, que la photographie soit dorénavant numérique, plutôt que d'en faire un médium indécis, fait qu'elle est connectée, qu'elle peut s'agréger à d'autres médiums, et qu'elle porte des métadonnées en quelque sorte chronogéographiques permettant à la fois de la géolocaliser et de connaître le moment exact de sa prise, ce qui s'avère utile pour certains travaux comme ceux d'Andreas Rutkauskas, tel que nous allons le voir.

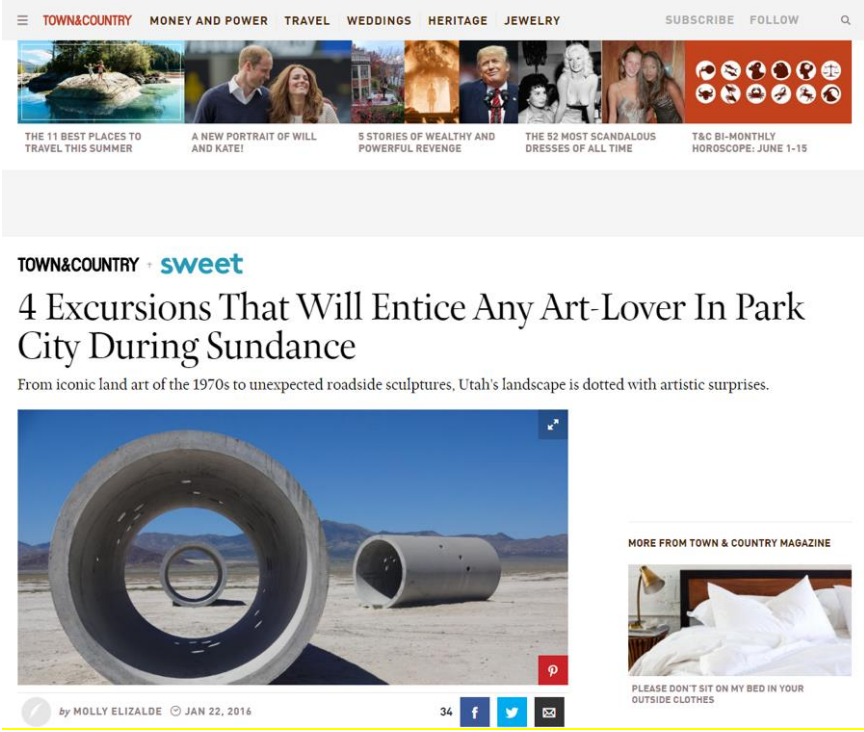

Figure 4: Page de la revue Town Eீ Country. Capture d'écran : https://www.townandcountrymag.com/leisure/travel-guide/news/a4842/art-related-sidetrips-to-take-from-park-city-ut-during-sundance/.

33 Pierre Bourdieu: Un art moyen. Essai sur les usages sociaux de la photographie, Paris, Éditions de Minuit, 1965. Voir à ce sujet : Suzanne Paquet, « Photographies de l'Autre, des nôtres, des autres. De la persistance de quelques usages sociaux », Captures, Figures, théories et pratiques de l'imaginaire, vol. $\mathrm{I}, \mathrm{n}^{\circ} \mathrm{I}$, mai 2016, http://revuecaptures.org/article-dunepublication/photographies-de-1\%E2\%80\%99autre-des-n\% 3 \% $\% \mathrm{~B}_{4}$ tres-des-autres (consulté le I8 mars 2019).

34 Michelle Henning, «New Lamps for Old: Photography, Obsolescence and Social Change $\gg$, Charles R. Acland (ed.), Residual media, Minneapolis, University of Minnesota Press, 2007, p. 48-65. Notre traduction. 
À travers la grande frénésie de documentation par l'image qu'entraîne la vie dans/par les réseaux sociaux, les photographies de sites (et aussi les photographies d'œuvres d'art ${ }^{35}$ ) circulent abondamment, comme autant d'invitations au voyage. La photographie et le tourisme - qui vont de pair et font même partie des grandes « inventions 》 du $19^{\mathrm{e}}$ siècle — étant des loisirs largement pratiqués au $2 \mathrm{I}^{\mathrm{e}}$ siècle, il faut trouver des destinations de plus en plus singulières, et les œuvres de land art semblent en être. La documentation, assurant toujours la survie des œuvres, serait devenue le fait des (photographes) amateurs qui donnent à celles-ci des parcours de vie inédits. C'est ainsi que les \#spiraljetty ou \#suntunnels font des milliers d'entrées sur Instagram, se partagent et «s'aiment» sur toutes les plateformes d'images photographiques. C'est ainsi également que des photographies d'amateurs sont utilisées sur des sites Web voués au tourisme afin d'encourager la visite de ces œuvres (voir la figure 4), sous des prétextes culturels ou d'économie régionale. Les œuvres de land art monumentales et pérennes, devenues intemporelles au même titre que les grands « monuments naturels » étatsuniens, sont perpétuellement actualisées par ces photographies mises en circulation dont le nombre augmente sans cesse, une forme d'hypercontemporanéité répondant, par l'accumulation des images, au caractère intemporel de ces nouveaux monuments. Ces mouvements, entre une réalité physique et sa représentation photographique, elle-même facteur concret de la vie des «Instagrammers ${ }^{36}$ » et autres internautes, façonnent aujourd'hui des modes d'action de plus en plus répandus ${ }^{37}$, contribuant (peut-être) à une meilleure connaissance de travaux désormais canoniques et accentuant ce que certains ont

${ }^{35}$ Nous sommes à l'ère de « l'art instagrammable », ne l'oublions pas... Voir, notamment, Dan Piepenbring, « Your Art's Not Instagrammable Enough, and Other News », The Paris Review, 22 juin 2017, https://www.theparisreview.org/blog/2017/06/22/your-arts-notinstagrammable-enough-and-other-news/ (consulté le I8 mars 20I9).

${ }^{36} \mathrm{Lev}$ Manovich, Instagram and the Contemporary Image, 2016, voir le site de l'auteur, http://manovich.net/index.php/projects/instagram-and-contemporary-image (consulté le is mars 2019).

37 «...the image is finally recognized for what it has always been: a representation of interconnected physical and social relations, and a system of informational relations (spatial and temporal) among people and the objects of their world » et « the once separated world of autonomous images and reality are technologically, functionally, perceptually and socially reconciled ». Francesco Lapenta, « Geomedia: On location-based media, the changing status of collective image production and the emergence of social-navigation systems », Visual Studies, vol. 26, n I, 20II, p. I6-17. 
qualifié d'épaississement du présent ${ }^{38}$. Cet épaississement se produirait, précisément, par la continuelle actualisation des images - et, du coup, de ce qu'elles montrent ou contiennent - actualisation générée par cumul ou entassement, les photographies d'un même motif se chassant constamment les unes les autres. Les images, à la fois fixes et en mouvement, composent la réalité de sites suffisamment figés pour faire eux-mêmes office de photographies.

\section{RES(T)ITUER (TEMPS ET LIEUX)}

Une forme de voyage, similaire, mais vers des images plutôt que vers des objets d'art cette fois, est pratiquée par Andreas Rutkauskas dans sa série Virtually There (voir la figure 5). Depuis 2009, l'artiste capte des images sur Google Earth, qui contient, on le sait, des photographies assemblées entre elles à travers lesquelles on peut naviguer, ce qui donne une impression de mouvement, de véritable déplacement. Google Earth serait ainsi l'instrument privilégié du voyage en fauteuil $\mathrm{du} 2 \mathrm{I}^{\mathrm{e}}$ siècle, une image technique en ayant remplacé une autre, l'activité restant très similaire. Et, tout comme le « voyageur casanier ${ }^{39} » d u{ }^{e} 9^{\mathrm{e}}$ siècle consentait parfois à quitter son fauteuil pour se mettre en mouvement, Rutkauskas, ayant accumulé des données, géographiques et autres, et noté les coordonnées spatiales des lieux « photographiés », s'y rend, « reenact[ing] the virtual journeys in real life », dit-iliº. Ayant effectué des captures d'écran sur Google Earth, il va sur les lieux pour les photographier à nouveau, à l'identique, ce qui nécessite une soigneuse géolocalisation afin d'arriver à correspondre à l'image prélevée. Au-delà de la réflexion sur le réel et le virtuel, il y a, dans cette série, une pensée forte sur le temps et l'espace tels qu'ils se vivent aujourd'hui.

\footnotetext{
${ }^{38}$ Entre autres, Nadav Hochman et Lev Manovich, « Zooming into an Instagram City: Reading the local through social media », First Monday, vol. $18, \mathrm{n}^{\mathrm{0}} 7$, juillet 2013, http://firstmonday.org/ojs/index.php/fm/article/view/47II/3698 (consulté le I8 mars 2019); Amelia Groom (ed.), Time, Londres, Whitechapel Gallery \& Cambridge, MIT Press coll. «Documents of Contemporary Art », 2013, p. 16.

${ }^{39}$ Pierre Bayard, Comment parler des lieux où l'on a pas été?, Paris, Éditions de Minuit, 20I2, p. I5.

40 Texte de présentation sur le site de l'artiste: http://www.andreasrutkauskas.com/virtually-there (consulté le I8 mars 2019).
} 

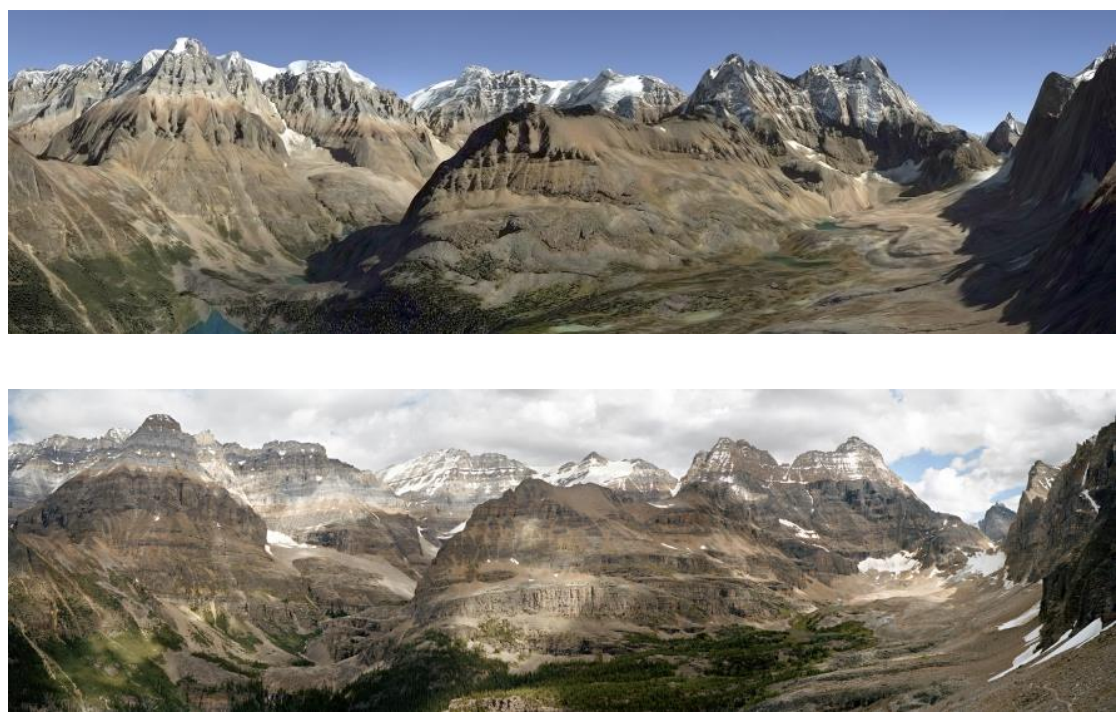

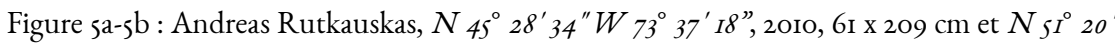
$5 I^{\prime \prime} W I I 6^{\circ} 20^{\prime} 3 I^{\prime \prime}, 2010,6 \mathrm{I} \times 209 \mathrm{~cm}$. De la série Virtually There. Avec l'aimable autorisation de l'artiste.

Virtually There semble res(t)ituer le temps pour diverses raisons, ou de diverses façons. D’abord, parce que les photographies de la série font appel à toute une tradition, celle de la photographie de paysage, et aussi parce qu'il s'agit de rephotographie (《reenactment», dit Rutkauskas); ensuite, parce que, pour qu'elles existent, l'artiste doit faire travailler ensemble des outils techniques datant d'époques éloignées les unes des autres. Au lieu d'un épaississement du présent, on assisterait plutôt ici à une (re)mise en place des photographies, à la superposition de zones temporelles - ce qui revient peut-être au même.

Pour produire la série, Rutkauskas a donc fait appel d'abord à des outils numériques, Internet, instruments de géolocalisation, de prélèvements d'images, etc., bref à des technologies de mise au point récentes et connectées, permettant l'accumulation et la mise en relation de données et, plus tard, le déplacement vers le site (réel) de l'image. Il a donc fait usage de la panoplie de l'explorateur contemporain connecté. Une fois sur les lieux, en revanche, l'artiste fait usage d'une vieille 
technique, la photographie à la chambre grand format ${ }^{4 \mathrm{I}}$, qui viendra redonner aux paysages une réalité temporelle, au sens de ce «qui est du domaine des choses matérielles ${ }^{42} \gg$, puisque l'instrument en est analogique, donc matériel au sens propre. L'utilisation de la chambre associe la série Virtually There aux photographies d'exploration d'il y a deux siècles, alors que les photographes bravaient territoires et éléments hostiles pour révéler et produire l'image d'un monde jusque-là peu ou mal connu. Des photographies qui, bien que commandées à des fins géographiques ou géologiques, donc scientifiques et éventuellement idéologiques, ont tout de même largement contribué à établir la grande tradition du paysage en photographie.

Retourner sur les lieux d'images déjà faites à la manière dont Rutkauskas le fait pour sa série répond à une autre tradition, celle de la re-photographie, mise à l'honneur à la fin des années $1970{ }^{43}$. Cet exercice veut que des séquences photographiques soient faites des mêmes endroits, photographiés de façon rigoureusement identique à des intervalles de temps réguliers, afin d'en surveiller les transformations. Dans le cas de Rutkauskas, il s'agirait d'une sorte de re-photographie à rebours, puisqu'elle est effectuée à une distance temporelle qui n'est pas vraiment significative et qu'il reprend la photographie avec un équipement qui préexistait, et depuis longtemps, à tout ce que Google (et ses exécutants) peut utiliser de matériel de prise de vues. Ce qui va à l'inverse de la re-photographie, qui se veut une manière de faire résolument « évolutive » en ce qu'elle marque le passage du temps en séries bien ordonnées, rendant visibles les modifications, détériorations ou restaurations des lieux et paysages. Ainsi, tout en faisant se correspondre des situations spatiales (de site Web en destination ou site physique) par différents moyens techniques, les images de Rutkauskas participent de quelques strates temporelles additionnées, qu'elles signalent en même temps.

La photographie est ainsi (motif de) mise en mouvement parce qu'elle est ellemême mobile, facile d'exécution et vouée à une large diffusion, d'abord par sa légèreté, puis par la multiplication des moyens sociotechniques propres au

\footnotetext{
${ }^{4 \mathrm{I}}$ Ces chambres sont des appareils photographiques aptes à produire de grands négatifs, de $4 \times 5$ pouces ou de $8 \times$ ro pouces généralement, demandant un travail minutieux et donnant des résultats photographiques d'une grande finesse.

${ }^{42}$ Le Grand Robert, https://gr.bvdep.com/robert.asp [référence non disponible pour les non-abonnés].

${ }^{43}$ Mark Klett (dir.), Second View. The Rephotographic Survey Project, Albuquerque, University of Mexico Press, 1984. Pour cette mission (1977), des paysages saisis par les «grands » du $19^{\mathrm{e}}$ siècle, O’Sullivan, Jackson, Hillers, Russell et Gardner, ont été rephotographiés cent ans plus tard par Klett et deux collègues.
} 
numérique et aux cultures qu'il a fait naître. Les deux types d'œuvres ici décrites, le land art et ses documents d'un côté, et, de l'autre, une photographie en quelque sorte performative et basée sur une documentation désormais accessible au plus grand nombre (les images fournies par Google), montrent que la quête géographique, celle du paysage, ou celle du lieu, reste d'actualité. Si cette quête s'est, depuis le $19^{\mathrm{e}}$ siècle, appuyée sur le couple mobilité et photographie, elle s'élargit maintenant au Web, qui ferait lui-même l'objet d'une spatialité à laquelle les temps de l'image s'additionneraient pour créer une chronogéographie permettant la circulation entre cyberespace et espace réel sans solution de continuité. Alors que la Spiral Jetty et les Sun Tunnels sont livrés aux amateurs, grâce à la production de nouveaux documents photographiques, et qu'une technologie propice au voyage casanier sert de point de départ à la série Virtually There, le temps des œuvres se fait fluide et connecté.

La série de Rutkauskas, bien qu'elle porte un titre appelant une pensée spatiale, présente des aspects chronologique et historique importants, en plus de rappeler que la photographie, parce qu'elle est un médium mobile, peut se considérer comme performative; tous ces éléments, assemblés, confèrent à l'œuvre un subtil feuilletage temporel. Virtually There compose tout à la fois une ouvre d'art et la documentation de bien étranges voyages, dont le motif de départ (le mobile comme le sujet au sens iconographique) n'est qu'un assemblage numérique que l'on met en mouvement par un pointeur ou une série de clics. Si elles semblent artificielles, les images de Google Earth ont pourtant le pouvoir, au moins pour l'artiste, d'appeler au déplacement, à l'action, qui devient parcours temporel et géographique, ce voyage effectué étant assimilable à celui qui mène désormais vers les œuvres de land art. Et les paysages visités et dépeints par Rutkauskas, à la suite de bien des explorateurs depuis bientôt deux siècles, semblent aussi immuables ou intemporels que les œuvres de land art. La série, amorcée en 2009, n'a pas trouvé sa fin. Elle reste en suspens, l'artiste retournant, ou étant susceptible de retourner, sur les lieux d'abord visités sur Google Earth, en une série d'allers et retours, une boucle par laquelle tout se répète (et se répétera) indéfiniment, en un itinéraire spatialement fermé sur lui-même — des coordonnées prélevées sur Google aux lieux physiques —, mais à la durée extensible, compressible, ou indifférente.

\section{CONTEMPORANÉITÉ}

Toute cette mobilité, cette dissémination des re-présentations des œuvres de Smithson et Holt, ou des images photographiques de Rutkauskas et des lieux qu'elles documentent, passe par des photographies devenues actives, performatives, dans la 
mesure où elles engagent à des déplacements : voyage vers les sites, (re)transmission d'images et voyages vers des images, toujours renouvelés. Par cette activité constante, c'est un présent et une présence en quelque sorte perpétuels qui s'imposent, c'est une capacité de passer d'un espace à l'autre, de l'espace matériel et physique à l'espace des flux d'images, et inversement. Pour décrire un présent continu des photographies ou des œuvres d'art actuel, on a parlé d'un devenir signalétique de l'image ${ }^{44}$, alors que les écrans ou les projections électroniques sont devenus omniprésents, entraînant un rafraîchissement ininterrompu de l'image numérique par balayage, le signal vidéo étant constant. La notion d'actualisation répétée de l'image, par apports successifs ou addition, les photographies s'accumulant et se chassant les unes les autres, semble mieux rendre compte d'un présent feuilleté des œuvres, passant par un travail collectif et la simultanéité ou le caractère consécutif des images, ce qui signale mieux leur ubiquité et leur contemporanéité. Contemporanéité que l’on pourrait illustrer ainsi :

Dans le geste même par lequel son présent divise le temps selon un « ne plus » et un « pas encore », elle instaure avec ces « autres temps » - certainement avec le passé et peut-être avec le futur — une relation particulière. Elle peut donc « citer » et, de cette manière, réactualiser un moment quelconque du passé4s.

Intégrant les flux numériques, la médialité des œuvres d'art aujourd'hui se fait fluctuante, ou variable: « [b] ecause of their mutability and multimediality, which are consequences of both what the technologies enable and what people do with them, digital cultural objects are inherently unstable ${ }^{46} \gg$. Les ouvres de land art, même plus anciennes que celles d'un Rutkauskas, n'y échappent pas. Les gestes, les voyages, l'expérience temporelle des sites — physiques et numériques - , une certaine matérialité des objets (s'il y en a), les documents - qu'ils servent de point de départ, d'intermédiaires ou de point d'arrivée - , leur circulation et ceux et celles qui s'en chargent, de même que les réseaux en tous genres, forment des complexes

44 Ingrid Hölzl, «The Photographic Now: David Claerbout's Vietnam », Intermédialités, $\mathrm{n}^{\circ}{ }_{17}$, « reproduire », 20II, https://www.erudit.org/fr/revues/im/20II-nI7imi817262/1005753ar/ (consulté le I8 mars 2019); Mette Sandbye, « It Has Not Been, It Is. The Signaletic Transformation of Photography », Journal of Aesthetics E\& Culture, vol. 4, $\mathrm{n}^{\circ} \mathrm{I}$, 20I2, http://www.tandfonline.com/doi/abs/10.3402/jac.v4io.I8I59 (consulté le I8 mars 2019)

${ }^{45}$ Agamben, 2008, p. 3I-32.

${ }^{46}$ Gillian Rose, «Rethinking the geographies of cultural "objects" through digital technologies: interface, network and friction », Progress in Human Geography, vol. 40, $n^{\circ} 3$, 2016, p. 339. En ligne : https://oro.open.ac.uk/43319/3/digital\%2oand\%2ocultural\%2ov4.pdf (consulté le I9 mars 2019). 
sociotechniques dans lesquels chacun des sites, chacun des moments, est essentiel. Et lorsqu'elles agissent avec (ou à même) ces œuvres d'art à médialité variable, les images photographiques, encore d'actualité bien qu'on ait voulu croire à leur tombée en désuétude ou à leur disparition, les res $(\mathrm{t})$ ituent, c'est-à-dire les rendent présentes, dans la contemporanéité des multiples sites, au regardeur. Assurément, la spatiotemporalité numérique et une chronogéographie des œuvres ne sauraient se concevoir sans le flux des images, leur circulation et les réseaux qui, par elles, se forment. Les images deviennent ainsi autant d'interfaces, de points de passage ou de points de bascule nécessaires entre des temps et des espaces qui auraient pu sembler trop éloignés ou incompatibles, créant la contemporanéité entre les temps de l'œuvre et ceux de sa réception. 


\title{
Du document aux flux : sites et temporalité(s) de quelques œuvres d'art
}

\author{
Suzanne Paquet, Université de Montréal
}

\section{RÉSUMÉ}

Cet article étudie, à partir de l'image (photographique), les modes d'être de certaines œuvres d'art, pour lesquelles il n'y a plus de médium unique, mais tout un réseau de gestes, d'images, de résonances et de reprises, circulant et se multipliant, composant ainsi une chronogéographie inédite. Ce sont les questions de leur médialité et des flux sociotechniques contribuant à faire de ces propositions artistiques des entités en réseaux, ainsi que leur temporalité particulière se conjuguant au présent et par la présence, qui sont examinées et exemplifiées à partir des œuvres Spiral Jetty (1970), de Robert Smithson, Sun Tunnels (1976), de Nancy Holt, et Virtually There (2009-), d'Andreas Rutkauskas.

\section{ABSTRACT}

Taking the photographic image as its starting point, this article examines artworks that exist not through a single medium, but through a complex network of movements, images, resonances, and repetitions, which circulate and multiply, opening up a peculiar chronogeography. Questions of mediality, temporality, and sociotechnical flux, which contribute to turning these artistic propositions into networked entities combining presence and the present (tense), are thus explored and exemplified through the artworks of Robert Smithson's Spiral Jetty (1970), Nancy Holt's Sun Tunnels (1976), and Andreas Rutkauskas's Virtually There (2009-).

\section{NOTE BIOGRAPHIQUE}

Suzanne Paquet est professeure agrégée au Département d'histoire de l'art et d'études cinématographiques de l'Université de Montréal. Ses domaines d'enseignement et de recherche sont les études photographiques et la sociologie de l'art. Ses textes ont été publiés dans différents ouvrages collectifs et dans des revues spécialisées, notamment les Cabiers de géographie du Québec, RACAR, Photoresearcher, la Nouvelle revue d'esthétique, Captures et Sens public. En 2009 paraissait son livre Le paysage façonné : les territoires postindustriels, l'art et l'usage aux Presses de l'Université Laval. Elle a également dirigé quelques ouvrages collectifs et numéros spéciaux de revues, dont le no I7, « reproduire », d'Intermédialités. 